\title{
Target selection and annotation for the structural genomics of the amidohydrolase and enolase superfamilies
}

\author{
Ursula Pieper · Ranyee Chiang · Jennifer J. Seffernick - Shoshana D. Brown • \\ Margaret E. Glasner · Libusha Kelly · Narayanan Eswar · J. Michael Sauder • \\ Jeffrey B. Bonanno $\cdot$ Subramanyam Swaminathan · Stephen K. Burley · \\ Xiaojing Zheng $\cdot$ Mark R. Chance $\cdot$ Steven C. Almo $\cdot$ John A. Gerlt $\cdot$ \\ Frank M. Raushel · Matthew P. Jacobson · Patricia C. Babbitt • \\ Andrej Sali
}

Received: 8 August 2008/Accepted: 12 December 2008/Published online: 14 February 2009

(C) The Author(s) 2009. This article is published with open access at Springerlink.com

\begin{abstract}
To study the substrate specificity of enzymes, we use the amidohydrolase and enolase superfamilies as model systems; members of these superfamilies share a common TIM barrel fold and catalyze a wide range of chemical reactions. Here, we describe a collaboration between the Enzyme Specificity Consortium (ENSPEC) and the New York SGX Research Center for Structural Genomics (NYSGXRC) that aims to maximize the structural coverage of the amidohydrolase and enolase superfamilies. Using sequence- and structure-based protein comparisons, we first selected 535 target proteins from a variety of genomes for high-throughput
\end{abstract}

Current affiliation of SGX Pharmaceuticals is Eli Lilly and Company, 10505 Roselle Street, San Diego, CA 92121, USA.

Electronic supplementary material The online version of this article (doi:10.1007/s10969-008-9056-5) contains supplementary material, which is available to authorized users.

\section{U. Pieper $(\bowtie)$}

Departments of Biopharmaceutical Sciences and Pharmaceutical Chemistry, California Institute for Quantitative Biosciences, University of California at San Francisco, Byers Hall at Mission Bay, Office 501-32, 1700 4th Street, San Francisco, CA 94158, USA e-mail: ursula@salilab.org

URL: http://salilab.org

R. Chiang · J. J. Seffernick · M. E. Glasner - L. Kelly ·

N. Eswar

Departments of Biopharmaceutical Sciences and Pharmaceutical Chemistry, California Institute for Quantitative Biosciences, University of California at San Francisco, Byers Hall at Mission Bay, Office 501, 1700 4th Street, San Francisco, CA 94158, USA

\section{S. D. Brown}

Department of Biopharmaceutical Sciences, California Institute for Quantitative Biosciences, University of California at San Francisco, Byers Hall at Mission Bay, Office 501C, 1700 4th Street, San Francisco, CA 94158, USA structure determination by X-ray crystallography; 63 of these targets were not previously annotated as superfamily members. To date, 20 unique amidohydrolase and 41 unique enolase structures have been determined, increasing the fraction of sequences in the two superfamilies that can be modeled based on at least $30 \%$ sequence identity from $45 \%$ to $73 \%$. We present case studies of proteins related to uronate isomerase (an amidohydrolase superfamily member) and mandelate racemase (an enolase superfamily member), to illustrate how this structure-focused approach can be used to generate hypotheses about sequence-structure-function relationships.

Keywords Amidohydrolase and enolase superfamilies . Structural genomics - Structure annotation .

Target selection

J. M. Sauder · S. K. Burley

SGX Pharmaceuticals Inc, 10505 Roselle Street, San Diego,

CA 92121, USA

J. B. Bonanno · S. C. Almo

Departments of Biochemistry and Physiology and Biophysics, Albert Einstein College of Medicine, Bronx, NY 10461, USA

S. Swaminathan

Biology Department, Brookhaven National Laboratory, Upton, NY 11973, USA

X. Zheng $\cdot$ M. R. Chance

Case Center for Proteomics \& Bioinformatics, Case Western

Reserve University, Cleveland, OH 44106, USA

J. A. Gerlt

Departments of Biochemistry and Chemistry,

University of Illinois, Urbana, IL 61801, USA 


\begin{tabular}{|c|c|}
\hline \multicolumn{2}{|c|}{ Abbreviations } \\
\hline PDB & Protein Data Bank \\
\hline NYSGXRC & $\begin{array}{l}\text { New York SGX Research Center for } \\
\text { Structural Genomics }\end{array}$ \\
\hline ENSPEC & Enzyme specificity consortium \\
\hline SFLD & Structure function linkage database \\
\hline PSI & Protein structure initiative \\
\hline NR & $\begin{array}{l}\text { Non-redundant database of protein } \\
\text { sequences }\end{array}$ \\
\hline I & Electrospray ionization \\
\hline IMM & Hidden Markov Model \\
\hline
\end{tabular}

\section{Introduction}

A long-standing challenge in biology is to predict the molecular function of proteins from their sequences and/or structures. This task is facilitated by a limited number of domain folds [1], restricting the set of structural types that must be studied in deducing a much larger set of functions. Special challenges, however, exist for functional prediction in different classes of proteins. For example, the function of an enzyme often cannot be correctly predicted because there are no clear links from the domain fold to the catalytic function and substrate specificity. Off-setting these problems, studies of genomes and sets of homologous proteins demonstrate that some aspects of catalysis are often conserved between evolutionarily-related proteins, even when these proteins catalyze different overall reactions [2-4]. This empirical observation restricts the functional space that must be considered, further

\section{F. M. Raushel}

Department of Chemistry, Texas A\&M University, P.O. Box 30012, College Station, TX 77842-3012, USA

\section{P. Jacobson}

Departments of Biopharmaceutical Sciences and Pharmaceutical Chemistry, California Institute for Quantitative Biosciences, University of California at San Francisco, 600 16th St., Box 2240, Genentech Hall at Mission Bay, Room N472C, San Francisco, CA 94158-2517, USA

\section{P. C. Babbitt}

Departments of Biopharmaceutical Sciences and Pharmaceutical Chemistry, California Institute for Quantitative Biosciences, University of California at San Francisco, Byers Hall at Mission Bay, Office 508E, 1700 4th Street, San Francisco, CA 94158, USA

\author{
A. Sali $(\square)$ \\ Departments of Biopharmaceutical Sciences and Pharmaceutical \\ Chemistry, California Institute for Quantitative Biosciences, \\ University of California at San Francisco, Byers Hall at Mission \\ Bay, Office 503B, 1700 4th Street, San Francisco, \\ CA 94158, USA \\ e-mail: sali@salilab.org \\ URL: http://salilab.org
}

facilitating prediction and leading to definitions of homologous sets of enzymes in terms of protein superfamilies and families based not only on structural conservation, but also on functional conservation [5]: Superfamily members share a common ancestor and potentially some aspects of function, while members of the same family are isofunctional, catalyzing the same overall reaction(s).

The large and diverse amidohydrolase and enolase superfamilies provide a particularly attractive opportunity to study the problem of predicting substrate specificity and enzymatic mechanisms from evolutionary and physical perspectives. These superfamilies are attractive targets because significant knowledge about the specificity of many of their members already exists, while there are still large areas of their sequence space where we do not have any structural or functional information.

Members of the amidohydrolase superfamily catalyze the hydrolysis of a wide range of substrates bearing amide or ester functional groups at carbon and phosphorus centers $[6,7]$. A common feature for this superfamily is a mononuclear or binuclear metal center coordinated in a $(\beta / \alpha)_{8}$-barrel (TIM barrel) polypeptide chain fold. The active site is formed by loops at the C-terminal ends of the $\beta$-strands. Currently, 36 named families have been identified based on the experimentally verified catalytic reactions. The set of superfamily sequences has been clustered into 90 subgroups based on sequence and in some cases active site similarities (the Structure-Function Linkage Database [8]: http://sfld.rbvi.ucsf.edu). In some subgroups, additional information about chemical reactions catalyzed by subgroup members is available; for many of the subgroups, however, no information about functional specificity is available.

Enolase superfamily members catalyze the abstraction of a proton $\alpha$ to a carboxylic acid to form an enolate anion intermediate $[9,10]$. Members of this superfamily share an $\mathrm{N}$-terminal $\alpha+\beta$ capping domain, as well as a $\mathrm{C}$-terminal $(\beta / \alpha)_{7} \beta$-barrel domain (modified TIM barrel). The active site is formed by loops at the C-terminal ends of the TIM barrel $\beta$-strands and two flexible loops from the capping domain; the active site also includes a $\mathrm{Mg}^{2+}$ ion [11]. Reactions catalyzed by enolases are less diverse than those of the amidohydrolases. The enolases are currently organized into 16 named families and 6 subgroups [8]. Approximately $50 \%$ of the sequences in the superfamily are of unknown function.

The amidohydrolase and enolase superfamilies are the focus of our Enzyme Specificity Consortium (ENSPEC), members of which include protein crystallographers, enzymologists, and computational biologists. We aim to predict the substrate specificity of an enzyme based on its experimentally determined and/or modeled structure 
$[2-4,7,10-42]$. This goal has been enabled by determination of crystallographic structures representing many of the amidohydrolase and enolase families.

To maximize the number of experimentally determined structures, ENSPEC has collaborated with the New York SGX Research Center for Structural Genomics (NYSGXRC), which is one of the four large-scale production centers of the Protein Structure Initiative (PSI) (http://www.nigms.nih.gov/ Initiatives/PSI; [43]). NIH guidelines mandate that $70 \%$ of the PSI targets come from diverse protein families selected by and shared among the four production centers [43]. About $15 \%$ of the targets are reserved for proteins of biomedical relevance defined by each center, and the remaining $15 \%$ are "community-nominated" targets. Several hundred of the NYSGXRC community targets are amidohydrolases and enolases nominated by ENSPEC. To date, the collaboration has determined 25 amidohydrolase and 50 enolase structures, contributing substantially to the total of 154 amidohydrolase and 89 enolase structures in the Protein Data Bank (PDB; 6/16/08) [44].

We begin by outlining the data sources and methods used for target selection and structure-based functional annotation (Materials and Methods). Second, we present the results of the target selection process, the status of the selected targets in the structural genomics pipeline, and the improvement in the modeling of the amidohydrolase and enolase superfamilies made possible by the new crystallographic structures (Results and Discussion). We conclude by discussing the biological impact of two sample target structures.

\section{Materials and methods}

Target selection

Target selection begins by identifying sequences of known members of the superfamilies (seed sequences), followed by filtering to obtain an initial target list. To identify additional members, we applied sequence- and structurebased expansion methods, followed by filtering for source organisms preferred by NYSGXRC. Superfamily membership for the additional targets was verified by expert curators by inspecting their sequences for probable catalytic residues. A web-based target selection tool was also constructed for further manual filtering to obtain the final target list.

\section{Seed sequence sources}

Verified amidohydrolase and enolase superfamily sequences (i.e., seed sequences) were obtained from the Structure Function Linkage Database (SFLD; http://sfld.rbvi.ucsf.edu/) [8].
The SFLD database is a manually constructed database that classifies enzymes hierarchically, based on specific sequence, structure, and functional criteria. The database is updated by a semi-automated method that detects new superfamily members by matching their sequences to Hidden Markov Models trained using the sequences of verified superfamily members, with subsequent manual inspection to verify the presence of catalytic residues. In June 2005, when our target list was constructed, the SFLD contained 3,701 amidohydrolases and 1,795 enolases. ${ }^{1}$

\section{Filtering of seed sequences}

PSI guidelines require that structural genomics targets share $\sim 30 \%$ or less amino acid sequence identity to a known theedimensional structure. To satisfy this condition, the seed amidohydrolase and enolase sequences were processed using the automated comparative modeling server MODWEB (http://salilab.org/modweb) [45]. Sequences with more than $30 \%$ sequence identity to any structure in the PDB over at least $70 \%$ of their length were identified and excluded from further consideration.

\section{Sequence-based expansion of amidohydrolase and enolase superfamily members}

For each seed amidohydrolase and enolase, homologous sequences in the UNIPROT database [46] were identified by the BUILD_PROFILE routine of MODELLER-9 [45]. BUILD_PROFILE is an iterative database-searching tool that relies on local dynamic programming to generate alignments and a robust estimate of their statistical significance. This method identified additional potential amidohydrolase and enolase sequences that were not present in the seed sequence pools.

Structure-based expansion of amidohydrolase superfamily members

In addition to the SFLD entries, we also used the known amidohydrolase superfamily structures to find additional potential amidohydrolase superfamily members (this expansion was not performed for the enolase superfamily). We began by splitting 100 PDB files containing known amidohydrolase structures (June 2005) into separate monomeric structures and clustering them at $80 \%$ sequence identity. The resulting 45 non-redundant structures were

\footnotetext{
1 The numbers of sequences in the publicly accessible version of the SFLD differ from those cited here because large numbers of sequences are undergoing curation at any given time and are therefore not yet listed on the public site.
} 
used for comparative modeling using the automated modeling server MODWEB [45].

First, each structure sequence was used as a query to find its homologs in UNIPROT using PSIBLAST [47]. Second, these homologs were modeled using the corresponding structure as a template. All models were deposited in our comprehensive MODBASE database of comparative protein structure models (http://salilab.org/modbase/; direct links to the datasets can be found in the supplemental materials) [48]. In addition, the amidohydrolase homologs found in UNIPROT were filtered by removing known amidohydrolase superfamily members, and then subjected to standard comparative modeling with MODWEB using all non-redundant chains in the PDB as potential templates. This step allowed us to eliminate sequences that are likely members of other superfamilies, judged by sequence identity and coverage.

\section{Filtering by organism}

While seed sequences could come from any genome, the additional amidohydrolase sequences identified by sequence- and structure-based expansions were filtered for ease of cloning to include only 79 organisms with genomic DNA available to NYSGXRC in 2005 and the marine metagenome from the Sargasso Sea sequencing project (formerly called environmental sequences) [49]. For simplicity, we call the 79 genomes plus the marine metagenome the "NYSGXRC genomes" (Table 1). The NYSGXRC reagent genomes have since been expanded to include over 115 organisms.

\section{Verification of catalytic residues}

The putative amidohydrolase sequences resulting from the sequence- and structure-based expansions were aligned to existing amidohydrolase Hidden Markov Models (HMMs) in the SFLD and manually inspected for probable catalytic residues. The final target list only includes sequences with at least $70 \%$ of the catalytic residues present.

\section{Target selection tool}

For final manual filtering of the target list, we constructed a web-based target selection tool. The tool comprises a combination of MySQL database tables with an interactive web-interface using LAMP [50]. It contains information about the sequences, including UNIPROT annotation, organism, sequence length, closest known structure, sequence identity to other cluster members, and domain boundaries for the TIM barrel domain obtained from SFLD. The interface allows searching for project datasets, organism groups, homologs based on sequence identity, and clusters of related sequences; the resulting sequences can be flagged for rejection or inclusion into the final target list.

Analysis of the target structures

The amidohydrolase and enolase superfamilies were annotated using computational tools. Cytoscape clustering gives an overview of how the targets are distributed across the superfamily [51]. Also, template-based modeling determines how many new sequences can be modeled with the new structural information [45].

\section{Sequence clustering of amidohydrolase superfamily by} cytoscape

The time required to perfom BLAST searches against the NCBI non-redundant database (NR) of protein sequences [52] was prohibitive due to the size and complexity of the superfamily. Thus, a custom database was created containing only the amidohydrolase sequences in the SFLD. To generate the all-by-all connections for cytoscape clustering, BLAST searches were then performed against this database at an E-value cutoff of $10^{-10}$, using each sequence in the set as a query. Because this custom database contained only sequences known to be members of the amidohydrolase superfamily, the generation of E-values is biased. Consequently, the E-values from this analysis cannot be directly compared to those calculated by BLAST against the NCBI NR database. A cytoscape [51] network was created from these BLAST results. In the absence of established statistical techniques for selecting the E-value cutoff, we examined the superfamily networks at a number of different E-value cutoffs, and present here only one of the corresponding networks, at an E-value cutoff of $10^{-10}$. Further discussion regarding choosing and interpreting Evalue cutoffs for sequence similarity networks may be found in [53]. Each node in the network represents a single sequence and each edge represents the pairwise connection between two sequences with the most significant BLAST E-value (better than the cut-off) connecting the two sequences. Lengths of edges are not meaningful, except that sequences in tightly clustered groups are more similar to each other than sequences with few connections. The nodes were arranged using the yFiles organic layout provided in Cytoscape version 2.4. Tools for visualization of protein networks were created by the UCSF Resource for Biocomputing, Visualization, and Informatics (http:// www.rbvi.ucsf.edu).

\section{Sequence clustering of enolase superfamily by cytoscape}

To generate the all-by-all connections for cytoscape clustering, BLAST analysis was performed against the NR 
database, using the sequences in the mandelate racemaselike, glucarate dehydratase-like, mannonate dehydrataselike, and muconate cycloisomerase-like subgroups of the SFLD enolase superfamily. The enolase subgroup was not included in this analysis. Almost all of the enolase subgroup members are in the enolase family, the sequences of which are all isofunctional, i.e. they all perform the wellcharacterized enolase reaction, important in glycolysis. Only hits in the aforementioned subgroups were used for further analysis. The cytoscape network was created as described above, but using an E-value cutoff for this superfamily of $10^{-40}$.

Table 1 List of 80 NYSGXRC genomes (as of June 2005)

\begin{tabular}{|c|c|c|c|}
\hline Organism & Taxonomy ID & Organism & Taxonomy ID \\
\hline Aeropyrum pernix & 56636 & Listeria monocytogenes & 1639 \\
\hline Aquifex aeolicus & 63363 & Metagenome sequences (Gene synthesis) & 256318 \\
\hline Arabidopsis thaliana & 3702 & Methanococcus jannaschi & 2190 \\
\hline Archaeoglobus fulgidus & 2234 & Mus musculus & 10090 \\
\hline Bacillus cereus & 1396 & Mycobacterium turberculosis $\mathrm{H} 37 \mathrm{Rv}$ & 83332 \\
\hline Bacillus halodurnas & 86665 & Mycoplasma pneumonia & 2104 \\
\hline Bacillus subtilis & 1423 & Neisseria gonorrhoeae & 485 \\
\hline Bacillus thuringiensis & 1428 & Neisseria meningitidis & 487 \\
\hline Bartonella henselae & 38323 & Nostoc & 1180 \\
\hline Bordetella pertussis & 520 & Oryctolagus cuniculus & 9986 \\
\hline Borrelia burgdorferi & 139 & Oryza sativa & 4530 \\
\hline Bos taurus & 9913 & Ovis aries & 9940 \\
\hline Caenorhabditis elegans & 6239 & Porphyromonas gingivalis & 837 \\
\hline Campylobacter jejuni & 197 & Pseudomonas aeruginosa & 287 \\
\hline Candida albicans & 5476 & Pseudomonas putida & 303 \\
\hline Canis familiaris & 9615 & Pyrococcus furiosus & 2261 \\
\hline Capra hircus & 9925 & Pyrococcus horikoshii & 53953 \\
\hline Caulobacter vibrioides & 155892 & Rattus norvegicus & 10116 \\
\hline Clostridium acetobutylicum & 1488 & Rhodobacter sphaeroides & 1063 \\
\hline Corynebacterium diphtheriae & 1717 & Saccharomyces cerevisiae & 4932 \\
\hline Cryptococcus neoformans & 5207 & Salmonella typhimurium & 602 \\
\hline Cryptosporidium parvum & 5807 & Schizosaccharomyces pombe & 4896 \\
\hline Deinococcus radiodurans & 1299 & Shigella Flexneri type 2a & 42897 \\
\hline Desulfovibrio vulgaris & 881 & Simian immunodeficiency virus & 11723 \\
\hline Dictyostelium discoideum & 44689 & Staphylococcus aureus & 1280 \\
\hline Drosophila melanogaster & 7227 & Staphylococcus epidermidis & 1282 \\
\hline Enterobacter cloacae & 550 & Streptococcus mutans & 1309 \\
\hline Enterococcus faecalis & 1351 & Streptococcus pneumoniae & 1313 \\
\hline Equus caballus & 9796 & Streptococcus pyogenes & 1314 \\
\hline Escherichia coli & 562 & Sulfolobus solfataricus & 2287 \\
\hline Escherichia coli $0157: \mathrm{H} 7$ & 83334 & Sus scrofa & 9823 \\
\hline Felis catus & 9685 & Takifugu rubripes & 31033 \\
\hline Gallus gallus & 9031 & Thermoplasma acidophilum & 2303 \\
\hline Haemophilus influenzae & 727 & Thermoplasma volcanium & 50339 \\
\hline Halobacterium sp. NRC-1 & 64091 & Thermotoga maritima & 2336 \\
\hline Helicobacter pylori & 210 & Ureaplasma urealyticum & 2130 \\
\hline Homo sapiens & 9606 & Vibrio cholerae & 666 \\
\hline Human immunodeficiency virus type 1 & 11676 & Xenopus laevis & 8355 \\
\hline Klebsiella pneumoniae & 573 & Xylella fastidiosa & 2371 \\
\hline Legionella pпеuторhila & 446 & Zea mays & 4577 \\
\hline
\end{tabular}




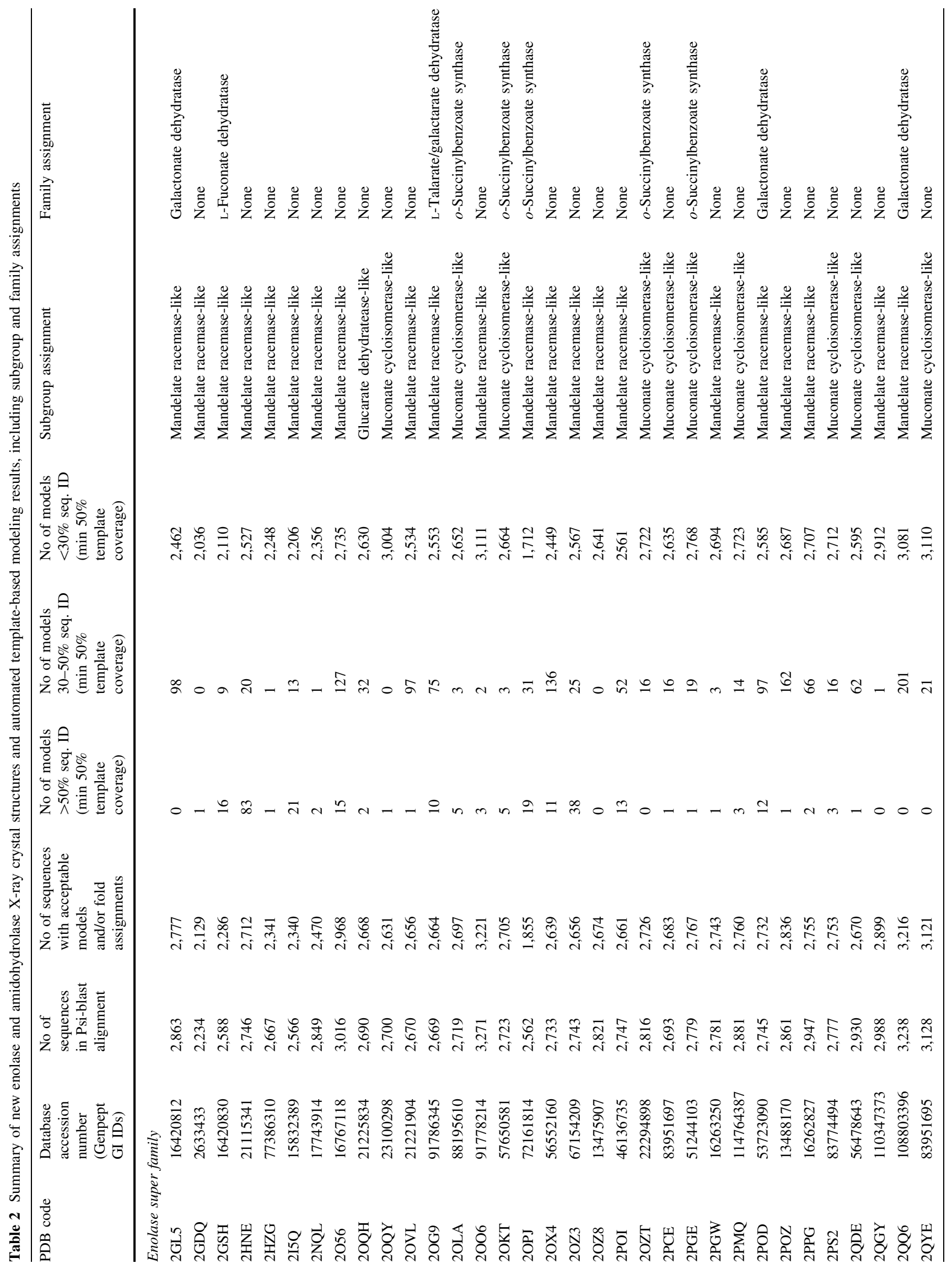




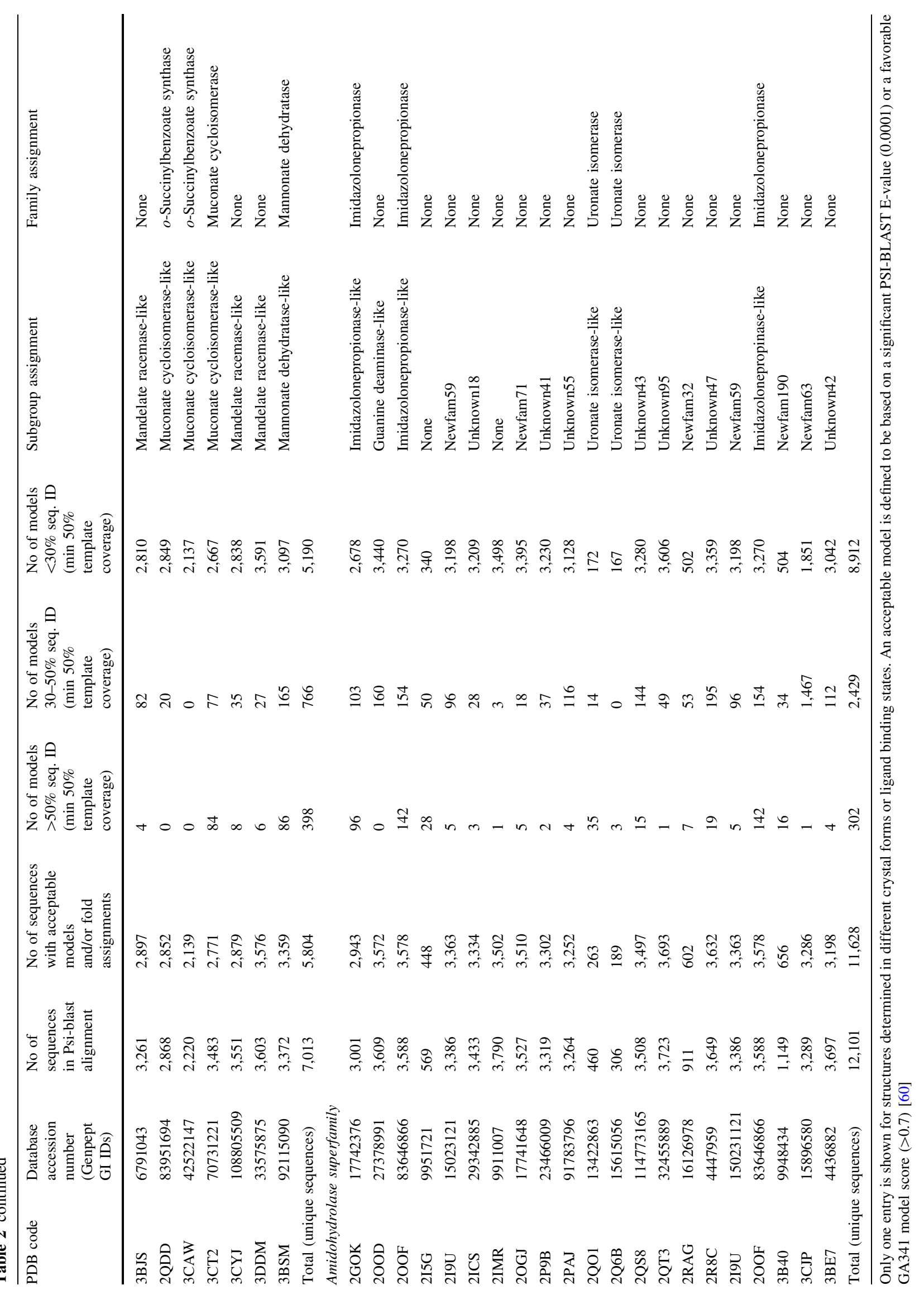




\section{Template-based modeling by MODWEB}

Automated comparative modeling of all known protein sequences using the new NYSGXRC crystallographic structures as templates was performed with MODWEB [45]. We relied on the MODWEB option that allows using a protein structure as input and results in models for all of the identifiable sequence homologs of the input structure from the NCBI NR database; these homologs were identified during ten PSI-BLAST iterations of the template sequence against NR (E-value cutoff is 0.0001). The results are available at http://salilab.org/modbase/models_nysgxrc_ latest.html (Table 2).

\section{Results and discussion}

We first present the results of the target selection procedure. We also describe the current snapshot of the progress of the targets through our structural genomics pipeline (June 2008). We then indicate how the resulting crystallographic structures are distributed across the two superfamilies. Next, we determine the number of protein sequences in the comprehensive sequence databases that are detectably related to these protein structures (i.e., the modeling leverage). Finally, for each of the two superfamilies, we describe an example target with interesting biological features.

\section{Target selection}

Given the capacities of ENSPEC and NYSGXRC, the goal was to identify approximately 500 target sequences, approximately evenly distributed between the two superfamilies. These targets were obtained by selecting representatives from previously identified superfamily members as well as by identifying new superfamily members in a select set of genomes (Materials and Methods).

\section{Targets for the amidohydrolase superfamily}

From the SFLD, we obtained a list of 3,701 amidohydrolase superfamily members. The first filtering step resulted in 1,918 sequences with less than $30 \%$ sequence identity to a known structure and at least 250 amino acid residues in length, originating from 424 organisms. We chose the $30 \%$ sequence identity limit, in congruence with NIH PSI guidelines, to concentrate our efforts on protein sequences with limited structural knowledge; sequences related at less than $30 \%$ sequence identity to the closest known structure are frequently modeled inaccurately due to errors in the corresponding target-template alignments [54-56].

These 1,918 sequences were further filtered manually using the target selection tool to obtain the reduced set of
224 target sequences. The selected amidohydrolase superfamily members are evenly distributed among the various clades of the superfamily, thus representing the diversity within the superfamily. Preference was given to the NYSGXRC genomes, but other organisms were also considered.

The 224 targets can be divided into 76 clusters with less than $30 \%$ sequence identity between any pair of sequences from two different clusters, 126 clusters at $50 \%$ sequence identity, and 177 clusters at $80 \%$ sequence identity. The amidohydrolase superfamily members all contain the defining conserved TIM barrel domain with some variation in their lengths; all targets are between 224 and 628 amino acid residues long, with $90 \%$ of them shorter than 500 residues. The length variation stems mostly from loops that connect the main secondary structure elements of the TIM barrel fold and is consistent with the previously observed size range for TIM barrel domains (150 to 500 residues [57]).

In addition to the known superfamily members, the sequence- and structure-based expansions detected 63 putative amidohydrolase superfamily members that were not initially in the SFLD (Table 3). These new potential targets fall into two categories: (i) divergent sequences that were detected by the sequence-based approach (Fig. 1, blue box) and (ii) divergent sequences that were detected by the structure-based approach (Fig. 1, orange box). Of the 63 putative amidohydrolase superfamily sequences, 50 were subsequently verified using the SFLD update procedure. The presence of probable catalytic residues for the remaining 13 targets was verified manually. Nine of these 13 sequences were detected by both the sequence- and structure-based approaches, and four sequences were only detected by the structure-based approach. Thus, the sequence- and structure-based approaches yielded 13 additional targets that could not be identified as amidohydrolase superfamily members using previously available protocols (corresponding to $21 \%$ of the new putative members of the amidohydrolase superfamily).

In summary, the final amidohydrolase target list includes 224 previously identified amidohydrolase superfamily members, as well as the 63 newly identified sequences. The final list includes 287 sequences from 53 organisms that cover $22(61 \%)$ of the named families in the superfamily (Fig. 2).

\section{Targets for the enolase superfamily}

We used a simpler selection scheme for the enolase superfamily members, because previous detailed studies have effectively found all of the superfamily members in publicly available sequence and structure databases (data not shown). Of the 1,795 sequences already established as enolase superfamily members, we selected as targets the 
Table 3 Putative amidohydrolase superfamily members

\begin{tabular}{|c|c|c|c|c|c|}
\hline $\begin{array}{l}\text { Database ID } \\
\text { (GenPept } \\
\text { GI IDs) }\end{array}$ & Method & Organism & Length & Annotation available at target selection & Verification \\
\hline 7462218 & Structure-based & Thermotoga maritima & 434 & Conserved hypothetical protein & HMM \\
\hline 7497374 & Structure-based & Caenorhabditis elegans & 818 & Hypothetical protein C44B7.10 & HMM \\
\hline 7500805 & Structure-based & Caenorhabditis elegans & 313 & $\begin{array}{l}\text { T21966 hypothetical protein } \\
\text { F38E11.3-Caenorhabditis elegans }\end{array}$ & HMM \\
\hline 9948434 & Structure-based & $\begin{array}{l}\text { Pseudomonas aeruginosa } \\
\quad \text { PAO1 }\end{array}$ & 448 & $\begin{array}{l}\text { Probable dipeptidase precursor (Pseudomonas } \\
\text { aeruginosa) }\end{array}$ & HMM \\
\hline 10173106 & Structure-based & Bacillus halodurans & 427 & BH0493 & HMM \\
\hline 10175729 & Structure-based & Bacillus halodurans & 571 & DNA-dependent DNA polymerase beta chain & HMM \\
\hline 13700943 & Structure-based & $\begin{array}{l}\text { Staphylococcus aureus } \\
\text { subsp. aureus N315 }\end{array}$ & 570 & DNA-dependent DNA polymerase beta chain & HMM \\
\hline 14600641 & Structure-based & Aeropyrum pernix & 313 & 313aa long hypothetical microsomal dipeptidase & HMM \\
\hline 14601853 & Template & Aeropyrum pernix & 394 & Hypothetical protein (Aeropyrum pernix) & HMM \\
\hline 14602106 & Structure-based & Aeropyrum pernix & 327 & Hypothetical protein (Aeropyrum pernix) & HMM \\
\hline 15600589 & Structure-based & $\begin{array}{l}\text { Pseudomonas aeruginosa } \\
\quad \text { PAO1 }\end{array}$ & 325 & $\begin{array}{l}\text { D82971 hypothetical protein PA5396 } \\
\text { (imported)_Pseudomonas aeruginosa (strain } \\
\text { PAO1) }\end{array}$ & HMM \\
\hline 15612748 & Structure-based & Bacillus halodurans & 448 & BH0185 & HMM \\
\hline 15614834 & Structure-based & Bacillus halodurans & 310 & Dipeptidase & HMM \\
\hline 15791917 & Structure-based & $\begin{array}{l}\text { Campylobacter jejuni } \\
\text { subsp. jejuni } \text { NCTC }\end{array}$ & 265 & Hypothetical protein Cj0556 & HMM \\
\hline 15805850 & Structure-based & $\begin{array}{l}\text { Deinococcus radiodurans } \\
\text { R1 }\end{array}$ & 418 & Hydrolase, putative & HMM \\
\hline 15896580 & Structure-based & Clostridium acetobutylicum & 262 & Predicted amidohydrolase (dihydroorotase family) & HMM \\
\hline 15898656 & Structure-based & Sulfolobus solfataricus & 314 & Microsomal dipeptidase & HMM \\
\hline 15925570 & Structure-based & $\begin{array}{l}\text { Staphylococcus aureus } \\
\text { subsp. aureus N315 }\end{array}$ & 336 & Conserved hypothetical protein & HMM \\
\hline 16125737 & Structure-based & Caulobacter vibrioides & 487 & $\begin{array}{l}\text { Uronate isomerase (EC 5.3.1.12) (Glucuronate } \\
\text { isomerase) (UronicDE isomerase) }\end{array}$ & HMM \\
\hline 16126978 & Structure-based & Caulobacter vibrioides & 417 & Dipeptidase & HMM \\
\hline 16127409 & Structure-based & Caulobacter vibrioides & 353 & Hypothetical protein & HMM \\
\hline 16130781 & Structure-based & Escherichia coli $\mathrm{K} 12$ & 464 & $\begin{array}{l}\text { Soluble protein involved in cell viability at the } \\
\text { beginning of stationary phase; soluble protein } \\
\text { involved in cell viability at the beginning of } \\
\text { stationary phase, contains urease domain }\end{array}$ & HMM \\
\hline 16410647 & Structure-based & $\begin{array}{l}\text { Listeria monocytogenes } \\
\text { EGD-e }\end{array}$ & 570 & $\operatorname{lmo} 1231$ & HMM \\
\hline 17556402 & Structure-based & Caenorhabditis elegans & 352 & Hypothetical protein Y71D11A.3a & HMM \\
\hline 19705473 & Structure-based & Rattus norvegicus & 336 & $\begin{array}{l}\text { 2-amino-3-carboxymuconate-6-semialdehyde } \\
\text { decarboxylase }\end{array}$ & HMM \\
\hline 19911227 & Structure-based & Homo sapiens & 336 & $\begin{array}{l}\text { 2-amino-3-carboxylmuconate-6-semialdehyde } \\
\text { decarboxylase }\end{array}$ & HMM \\
\hline 19911231 & Structure-based & Caenorhabditis elegans & 401 & $\begin{array}{l}\text { 2-amino-3-carboxylmuconate-6-semialdehyde } \\
\text { decarboxylase }\end{array}$ & HMM \\
\hline 24379660 & Structure-based & $\begin{array}{l}\text { Streptococcus mutans } \\
\text { UA159 }\end{array}$ & 267 & conserved hypothetical protein & HMM \\
\hline 33592291 & Structure-based & $\begin{array}{l}\text { Bordetella pertussis } \\
\text { Tohama I }\end{array}$ & 284 & $\begin{array}{l}\text { Putative 2-pyrone-4,6-dicarboxylic acid } \\
\text { hydrolase }\end{array}$ & HMM \\
\hline 33593502 & Structure-based & $\begin{array}{l}\text { Bordetella pertussis } \\
\text { Tohama I }\end{array}$ & 341 & Putative dipeptidase & HMM \\
\hline 39976001 & $\begin{array}{l}\text { Sequence- and } \\
\text { structure-based }\end{array}$ & Magnaporthe grisea $70-15$ & 417 & Hypothetical protein & HMM \\
\hline
\end{tabular}


Table 3 continued

\begin{tabular}{|c|c|c|c|c|c|}
\hline $\begin{array}{l}\text { Database ID } \\
\text { (GenPept } \\
\text { GI IDs) }\end{array}$ & Method & Organism & Length & Annotation available at target selection & Verification \\
\hline 42527610 & Structure-based & $\begin{array}{l}\text { Treponema denticola } \\
\quad \text { ATCC } 35405\end{array}$ & 371 & Dihydroorotase, putative & HMM \\
\hline 42631159 & Structure-based & Haemophilus influenzae & 330 & Hypothetical protein & HMM \\
\hline 51012913 & Structure-based & Saccharomyces cerevisiae & 313 & YMR262W & HMM \\
\hline 51968376 & Structure-based & Arabidopsis thaliana & 346 & Unnamed protein product & HMM \\
\hline 51968996 & Structure-based & Arabidopsis thaliana & 346 & Unnamed protein product & HMM \\
\hline 55980841 & Structure-based & Thermus thermophilus HB8 & 369 & Amidohydrolase family protein & HMM \\
\hline 60279993 & STRUCTURE-based & Pseudomonas aeruginosa & 403 & PvdM HMM & \\
\hline 66807941 & Structure-based & Dictyostelium discoideum & 359 & Hypothetical protein & HMM \\
\hline 66808659 & Structure-based & Dictyostelium discoideum & 322 & Hypothetical protein & HMM \\
\hline 1065989 & Sequence-based & $\begin{array}{l}\text { Bacillus subtilis subsp. } \\
\text { subtilis } \text { str. } 1\end{array}$ & 577 & Adenine deaminase & HMM \\
\hline 15023784 & Sequence-based & Clostridium acetobutylicum & 570 & Adenine deaminase & HMM \\
\hline 24636152 & Structure-based & Caenorhabditis elegans & 403 & Hypothetical protein C44B7.12 & HMM \\
\hline 29377069 & Structure-based & Enterococcus faecalis V583 & 444 & Chlorohydrolase family protein & HMM \\
\hline 40788915 & Structure-based & Homo sapiens & 777 & $\begin{array}{l}\text { Q93075_chr3:10265710- } \\
\text { 10295706_H233R_V272I_L374P PUTATIVE } \\
\text { DEOXYRIBONUCLEASE KIAA0218 } \\
\text { (EC 3.1.21.-) }\end{array}$ & HMM \\
\hline 45446932 & $\begin{array}{l}\text { Sequence- and } \\
\text { structure-based }\end{array}$ & Drosophila melanogaster & 774 & CG32626-PA, isoform A & HMM \\
\hline 56203368 & $\begin{array}{l}\text { Sequence- and } \\
\text { structure-based }\end{array}$ & Homo sapiens & 776 & $\begin{array}{l}\text { Adenosine monophosphate deaminase } 1 \\
\text { (isoform M }\end{array}$ & HMM \\
\hline 56203369 & Sequence-based & Homo sapiens & 780 & OTTHUMP00000059283 & HMM \\
\hline 57230710 & Structure-based & Filobasidiella neoformans & 469 & Hydrolase, putative & HMM \\
\hline 63055053 & Structure-based & Homo sapiens & 761 & TatD DNase domain containing 2 & HMM \\
\hline 68250266 & Structure-based & Haemophilus influenzae & 251 & Conserved putative deoxyribonuclease & HMM \\
\hline 429129 & Sequence-based & Saccharomyces cerevisiae & 797 & $\begin{array}{l}\text { YB9Z_YEAST HYPOTHETICAL } 92.9 \text { KD } \\
\text { PROTEIN IN SSH1-APE3 INTERGENIC } \\
\text { REGION }\end{array}$ & Manual \\
\hline 7293948 & Sequence-based & Drosophila melanogaster & 520 & CG5998-PA & Manual \\
\hline 11463854 & Sequence-based & Drosophila melanogaster & 561 & Male-specific IDGF & manual \\
\hline 14602062 & Structure-based & Aeropyrum pernix & 375 & Hypothetical protein [Aeropyrum pernix] & Manual \\
\hline 15898896 & Structure-based & Sulfolobus solfataricus & 269 & Conserved hypothetical protein & Manual \\
\hline 16264026 & Template & Sinorhizobium meliloti & 466 & HYPOTHETICAL PROTEIN & Manual \\
\hline 17646150 & $\begin{array}{l}\text { Sequence- and } \\
\text { structure-based }\end{array}$ & Drosophila melanogaster & 506 & Adenosine deaminase-related growth factor $\mathrm{C}$ & Manual \\
\hline 23093239 & Sequence-based & Drosophila melanogaster & 561 & CG32178-PA & Manual \\
\hline 25009707 & Sequence-based & Drosophila melanogaster & 561 & AT05468p & Manual \\
\hline 33593596 & Structure-based & $\begin{array}{l}\text { Bordetella pertussis } \\
\text { Tohama I }\end{array}$ & 523 & Conserved hypothetical protein & Manual \\
\hline 40744823 & Structure-based & $\begin{array}{l}\text { Aspergillus nidulans FGSC } \\
\text { A4 }\end{array}$ & 562 & HYPOTHETICAL protein & Manual \\
\hline 47678365 & Sequence-based & Homo sapiens & 511 & $\begin{array}{l}\text { Cat eye syndrome critical region } \\
\text { protein } 1 \text { [Homo sapiens] }\end{array}$ & Manual \\
\hline 49116836 & $\begin{array}{l}\text { Sequence- and } \\
\text { structure-based }\end{array}$ & Xenopus laevis & 510 & Hypothetical protein & Manual \\
\hline
\end{tabular}

Tables listing all amidohydrolase and enolase superfamily targets can be found at http://salilab.org/projects/enspec/ (HMM Hidden Markov Model verification) 


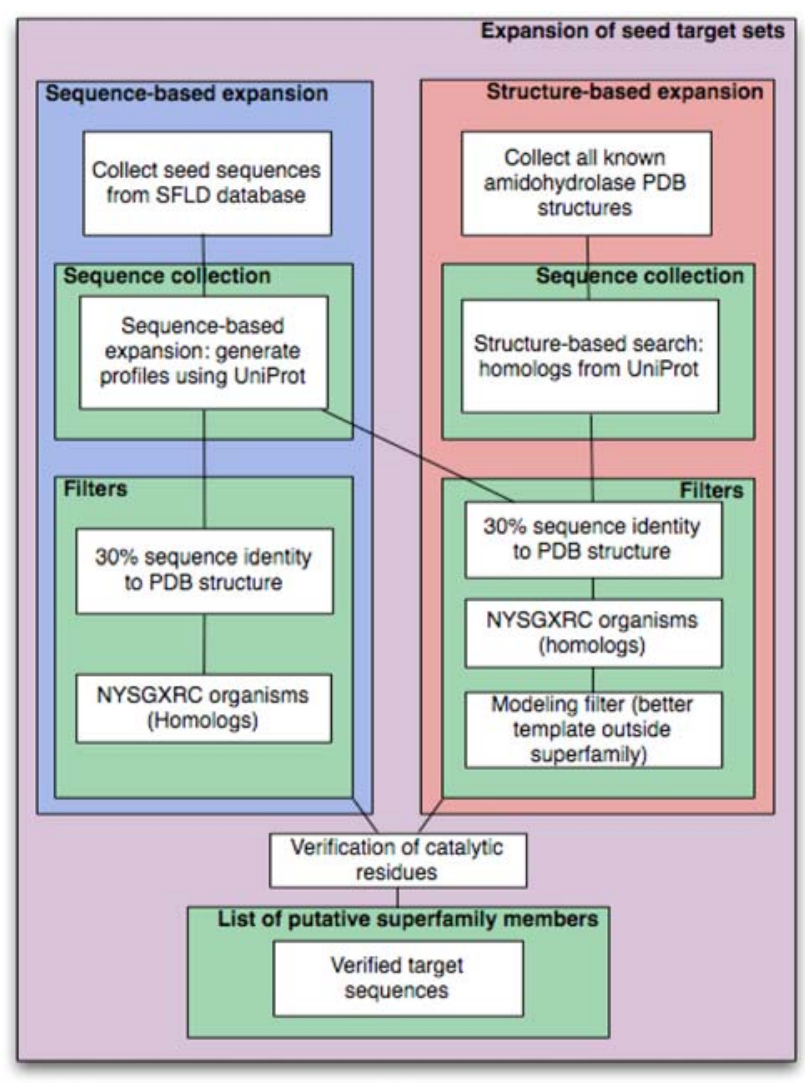

Fig. 1 Flowchart of the target expansion strategy of sequence-based target expansion (left) and structure-based target expansion (right)

255 sequences with less than $30 \%$ sequence identity to a known structure over at least 250 residues in length, originating from 98 organisms. These targets form 74 clusters at the $30 \%$ sequence identity cutoff, 126 clusters at $50 \%$ sequence identity, and 196 clusters at $80 \%$ sequence identity. The length distribution is 200 to 656 amino acid residues, with $90 \%$ of the sequences between 200 and 405 residues in length.

A complete list of the selected amidohydrolase and enolase superfamily targets can be found at http://salilab. org/projects/enspec/.

\section{Structural genomics pipeline attrition}

To date, 254 amidohydrolase (88\%) and 206 enolase (80\%) superfamily members have been attempted using the NYSGXRC/ENSPEC X-ray crystallographic structure determination pipeline. Progress to date and attrition rate at each stage of the pipeline are documented in Table 4 (June 2008). The project has not yet been completed, and a number of targets are still progressing through the pipeline. Also, a few targets in the target list have not yet been entered in the experimental pipeline. Therefore, the final overall success rate should be higher than that presented in
Table 4. Experimental results for all NYSGXRC Community-nominated targets are updated weekly in PepcDB (http://pepcdb.pdb.org/).

Clear trends are observed in the success rates of crystallization and subsequent crystallographic structure determination for the amidohydrolase and enolase superfamily members. While only $38 \%$ of the purified targets were members of the enolase superfamily, they comprise $67 \%$ of the unique experimental structures. If crystals are obtained for an enolase superfamily member, there is a good chance that its structure will be successfully determined. On the other hand, for at least a quarter of the amidohydrolase proteins, we observed unusually broad peaks in the electrospray ionization (ESI) mass spectra of the intact proteins, indicative of heterogeneity in the preparation. Proteolytic digestion followed by tandem mass spectrometry analysis was carried out on the heterogeneous proteins; multiple sites of oxidation and methylation were identified with $90 \%$ of the protein sequence typically identified. These modifications were the source of the sample heterogeneity, and thus one reason for the limited success in obtaining usable crystallographic datasets from crystals of these amidohydrolases.

Of structural and functional interest was the fact that the oxidation sites were primarily located at histidine residues adjacent to $\mathrm{Fe}^{2+}$ ions in the presumed active sites of the amidohydrolases. Excess oxidation can be avoided using an alternate expression system (e.g. baculovirus) or adding excess $\mathrm{Mn}^{2+}$ and an iron chelator such as $2,2^{\prime}$-dipyridyl prior to induction during $E$. coli expression. In contrast, oxidation was not been observed in members of the enolase superfamily, since these proteins bind only a divalent metal ion such as $\mathrm{Mg}^{2+}$ or $\mathrm{Mn}^{2+}$ and not iron.

\section{Analysis of the resulting crystallographic structures}

\section{Leverage of new crystallographic structures by modeling}

To determine the impact of a structure on the structural mapping of the protein sequence space, we determine how many known protein sequences can be modeled based on the structure (i.e., the modeling leverage) (Table 2). Each enolase structure is a useful template for calculating comparative models for 2,500 to 3,200 other protein sequences in the NR database; a template is considered useful when the resulting model is based on a significant PSI-BLAST E-value (0.0001) or a favorable GA341 model score $(>0.7)$. In contrast, the amidohydrolase superfamily structures fall into two categories: most are detectably related to 3,000-3,800 other proteins, but five structures (PDB Codes: 2I5G, 2Q01, 2Q6E, 2RAG, and 3B40) are related to a significantly smaller number of sequences (approximately 300-1,000). 
Fig. 2 Phylogenetic tree of the organisms for the selected amidohydrolase targets. The numbers in parentheses represent the number of targets for confirmed (first number) and putative (second number) amidohydrolase superfamily members. The tree was generated using the NCBI Taxonomy Browser [61]

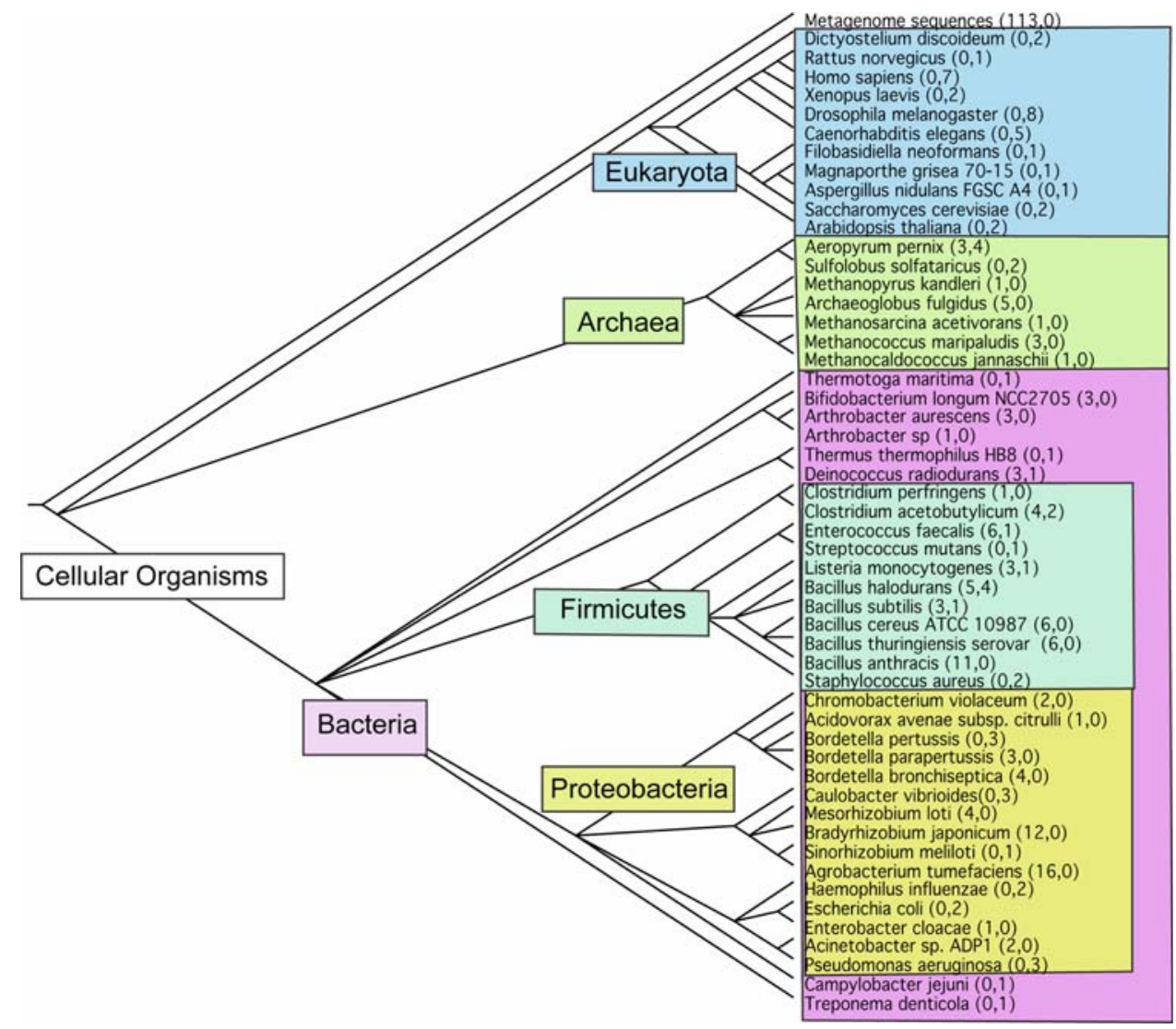

A comparison of these numbers to the template-based modeling results for all NYSGXRC structures as of May 2007 (Table 5) shows that the average number of models per structure is significantly higher for the amidohydrolase and enolase superfamilies than for all structures determined by NYSGXRC $(2,681$ vs. 1,964$)$. This difference reflects the relatively large sizes of the amidohydrolase and enolase

Table 4 Success rates for the steps in the structural genomics pipeline as of June 2008

\begin{tabular}{|c|c|c|c|c|c|c|}
\hline \multirow[t]{2}{*}{ Step } & \multicolumn{2}{|c|}{$\begin{array}{l}\text { Amidohydrolase } \\
\text { superfamily }\end{array}$} & \multicolumn{2}{|c|}{$\begin{array}{l}\text { Enolase } \\
\text { superfamily }\end{array}$} & \multicolumn{2}{|c|}{$\begin{array}{l}\text { Both } \\
\text { superfamilies }\end{array}$} \\
\hline & Total & $\begin{array}{l}\text { Fraction } \\
(\%)\end{array}$ & Total & $\begin{array}{l}\text { Fraction } \\
(\%)\end{array}$ & Total & $\begin{array}{l}\text { Fraction } \\
(\%)\end{array}$ \\
\hline In pipeline & 279 & & 222 & & 501 & \\
\hline Cloned & 254 & 91 & 206 & 93 & 460 & 92 \\
\hline Expressed & 225 & 88 & 177 & 86 & 402 & 87 \\
\hline Soluble & 167 & 74 & 112 & 63 & 279 & 69 \\
\hline Purified & 110 & 66 & 67 & 60 & 177 & 63 \\
\hline Crystallized & 63 & 57 & 44 & 66 & 107 & 60 \\
\hline $\begin{array}{l}\text { Unique } \\
\text { structures }\end{array}$ & 20 & 32 & 41 & 93 & 61 & 57 \\
\hline $\begin{array}{l}\text { All } \\
\text { structures }\end{array}$ & 25 & & 50 & & 75 & \\
\hline
\end{tabular}

superfamilies; according to the Superfamily database (http://supfam.org, [58]), across all of the superfamilies in the database, there are on average 1,770 protein sequences per superfamily.

Breaking down the modeling leverage by sequence identity reveals that the modeling leverage for the amidohydrolase and enolase superfamily structures is higher and lower than that for all NYSGXRC structures below and above the sequence identity cutoff of $30 \%$, respectively. These differences are likely due in part to the relatively high diversity in the amidohydrolase and enolase superfamilies.

Upon initiation of the ENSPEC/NYSGXRC effort in June 2005 , 45\% of all known members of the amidohydrolase and enolase superfamilies were related to a known structure with a sequence identity higher than $30 \%$. Due to the increased number of templates from the amidohydrolase and enolase superfamilies contributed by our consortia, this number increased to from $45 \%$ to $73 \%$.

The total number of unique sequences modeled using the new amidohydrolase and enolase superfamily structures is 11,097 , approximately $30 \%$ more than the number of known sequences from the amidohydrolase and enolase superfamilies. Among these additional sequences, we expect both members of other superfamilies with the TIM 
Table 5 Comparison of template-based modeling statistics for the 61 ENSPEC/NYSGXRC structures and all 327 NYSGXRC structures (May 2007)

\begin{tabular}{|c|c|c|}
\hline & $\begin{array}{l}\text { Amidohydrolase and enolase superfamily } \\
\text { members }\end{array}$ & All \\
\hline Average number of sequences with acceptable models & 2,681 & 1,964 \\
\hline Minimum/maximum number of sequences with acceptable models & $189 / 3693$ & $30 / 6320$ \\
\hline Average number of sequences with $>50 \%$ sequence identity, at least $50 \%$ coverage & 15 & 20 \\
\hline $\begin{array}{l}\text { Average number of sequences with } 30-50 \% \text { sequence identity, at least } 50 \% \\
\text { coverage }\end{array}$ & 59 & 113 \\
\hline Average number of sequences with $<30 \%$ sequence identity, at least $50 \%$ coverage & 2,572 & 1,400 \\
\hline
\end{tabular}

An acceptable model is defined to be based on a significant PSI-BLAST E-value (0.0001) or a favorable GA341 model score ( $>0.7$ )

barrel fold, as well as currently unidentified members of the amidohydrolase and enolase superfamilies, because the sequence databases have been growing by approximately $50 \%$ since 2005 , and also because we concentrated on selecting only targets from the NYSGXRC genomes in the target selection process for this project.

Distribution of targets over the amidohydrolase and enolase superfamilies

For large groups of related sequences, such as the amidohydrolase superfamily network-based visualization of their relationships is helpful in generating hypotheses about how various enzymes in the superfamily evolved, and on how closely the subgroups are related to each other. We have plotted cytoscape networks for the amidohydrolase and enolase superfamilies, based on clustering by sequence similarity, and marked previously known structures, and the final targets and the structures from this project (Fig. 3). For clarity, we circled a few distinct subgroups. Another network representation with all sub-group assignments can be found in the supplemental materials.

Many subgroups in the large amidohydrolase superfamily, such as the urease-like subgroup and the uronate isomerase-like subgroup, are distinctly separated from the other superfamily members. This separation can most simply be interpreted as the result of the extreme divergence of these subgroups; thus, they are "outliers" in the overall context of the superfamily (see below for further discussion of this subgroup).

Four of the five divergent amidohydrolase structures with a considerably smaller number of homologs are separated from the main amidohydrolase network, even at the relatively non-stringent E-value cut-off of $10^{-10}$ required to visualize connections between nodes. Two of them (2Q01, 2Q6E) belong to the uronate isomerase-like subgroup. Another two of these structures (2RAG, 3B40) are clustered together with a number of unclassified sequences as well as several membrane dipeptidase-like amidohydrolase superfamily members, possibly indicating that these targets are additional members of the membrane dipeptidase subgroup. This subgroup membership is also supported by their annotation as putative dipeptidases in UniProt.

For the enolase superfamily, we chose to generate a cytoscape network that represents only four subgroups, containing the majority of the targets. The targets were mostly chosen from the mandelate racemase-like subgroup, because it is the largest subgroup with little previous structural coverage, and from the more divergent muconate cycloisomerase subgroup. The cytoscape networks illustrate that the targets and the resulting structures are indeed concentrated in regions of superfamily sequence space that lacked structural characterization prior to the start of the project, as desired for our target selection.

Examples of biological impact resulting from new structures obtained in this study

\section{Amidohydrolase superfamily example: atypical uronate isomerase Bh0493}

The enzymes in the uronate isomerase family are members of the amidohydrolase superfamily, although they are extremely diverged from other clusters of the amidohydrolase superfamily network (Fig. 3a). Target 9247a (gi 10173106, Bh0493) from Bacillus halodurans was identified by our structure-based expansion as a putative member of the amidohydrolase superfamily and has recently been experimentally confirmed as a uronate isomerase [29]. In most organisms, both glucuronic acid and galacturonic acid are first isomerized by a single uronate isomerase, followed by further modification by several sugar specific dehydrogenases and dehydratases. In B. halodurans, as in several other organisms, two uronate isomerase genes are found, in 

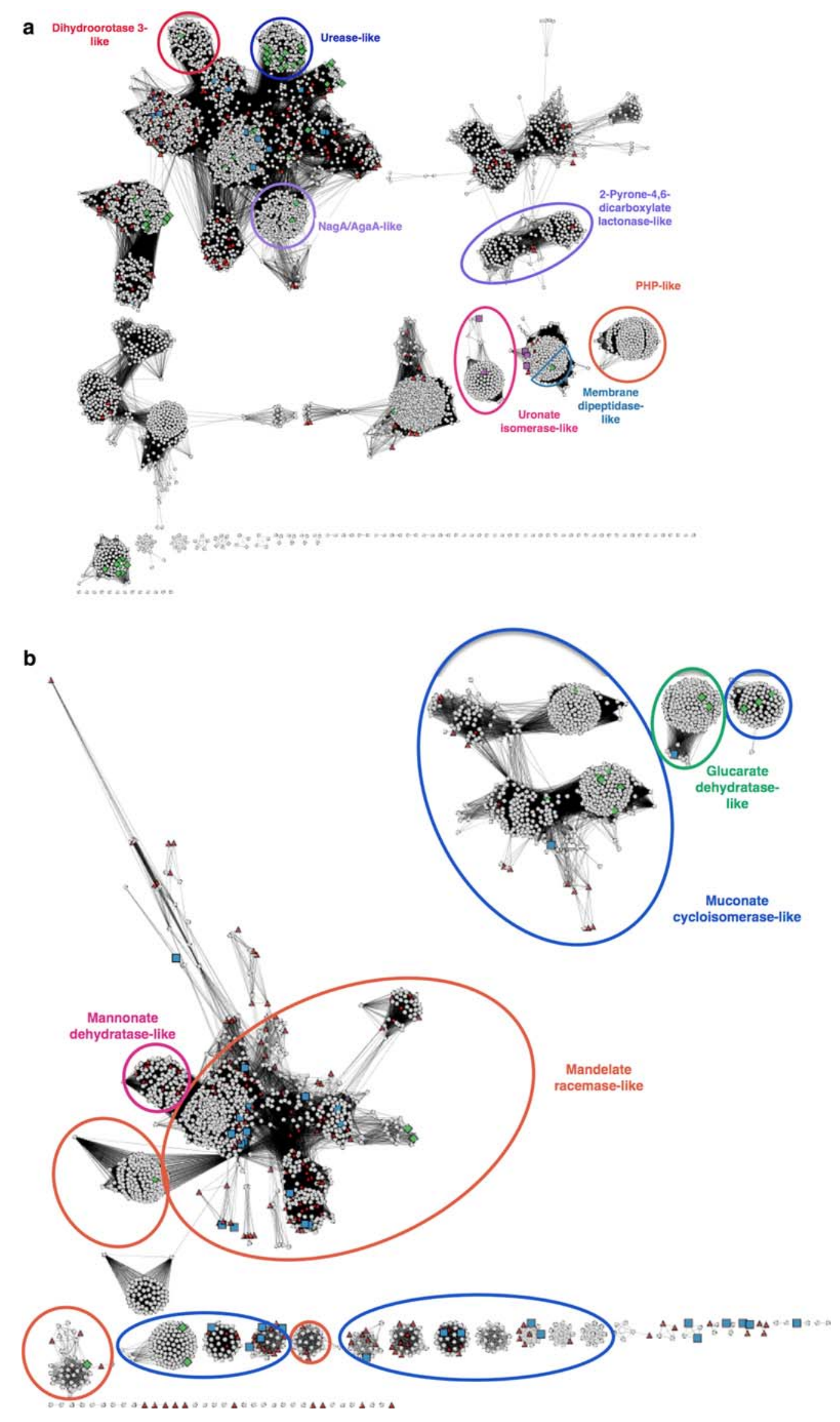
4 Fig. 3 a Cytoscape clustering for the amidohydrolase superfamily. The most homogeneous subgroups have been named. An additional figure with full subgroup coloring is available in Supplemental Materials. Green diamonds Structures determined prior to the start of the ENSPEC/NYSGXRC project in June 2005. Red triangles Superfamily members in the target list. Purple squares Five divergent structures determined by ENSPEC/NYSGXRC. Blue squares All other structures determined by ENSPEC/NYSGXRC. Ovals indicate subgroups: red dihydroorotase 3-like; dark blue urease-like; purple NagA/AgaA-like; light-blue: 2-Pyrone-4,6-dicarboxylate lactonaselike; pink uronate-isomerase-like; orange PHP-like; delft-blue membrane dipeptidase-like. b Cytoscape clustering for the enolase superfamily. Subgroup clusters are marked for four subgroups. The full subgroup assignments can be found in Supplemental Materials. Green diamonds Structures determined prior to the start of the ENSPEC/NYSGXRC project in June 2005. Red triangles Superfamily members in the target list. Blue squares All structures determined by ENSPEC/NYSGXRC. Ovals indicate subgroups: pink mannonate dehydratase-like; orange mandelate racemase-like; blue muconate cycloisomerase-like; green glucarate dehydratase-like operons containing dehydrogenase as well as dehydratase enzymes, consistent with this assignment of activity. We characterized both uronate isomerase genes, a "typical" uronate isomerase, Bh0705, and Bh0493, an "outlier" relative to other characterized members of this family (Fig. 4a). Although the results showed that each enzyme can isomerize both substrates, galacturonate and glucuronate, the Bh0705 uronate isomerase preferentially isomerizes glucuronic acid (approximately 100 times faster than galacturonic acid). In contrast, Bh0493 isomerizes glucuronic acid and galacturonic acid almost equally efficiently. These observations indicate that in B. halodurans, the "typical" uronate isomerase (Bh0705) has specialized its catalytic activity to preferentially isomerize glucuronic acid, perhaps because the isomerization of galacturonic acid is sufficiently achieved by Bh0493.
Fig. 4 a Cytoscape network showing the uronate isomerase family. The E-value threshold for displaying edges is $10^{-10}$. The large cluster represents the "typical" uronate isomerases; sequences in this cluster are more similar to other members of the amidohydrolase superfamily than is Bh0493. Bh0705 is shown in purple and the structurally characterized enzyme from Thermotoga maritima is shown in red. On the right, the outlier uronate isomerase, Bh0493, is shown in purple along with a small number of sequences of unknown function. b Ribbon diagram [62] of a superposition of the trimeric structures of Bh0493 (2Q6E, blue) and a uronate isomerase from Thermotoga maritima (1J5S, red). The detailed box shows the active site residues of chain $\mathrm{A}$ including a $\mathrm{Zn}^{2+}$ ion for 2Q6E

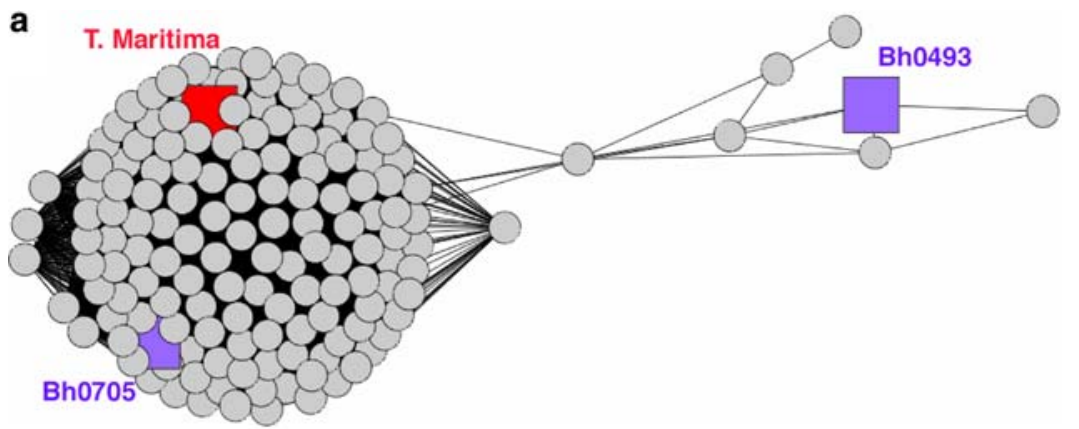

b

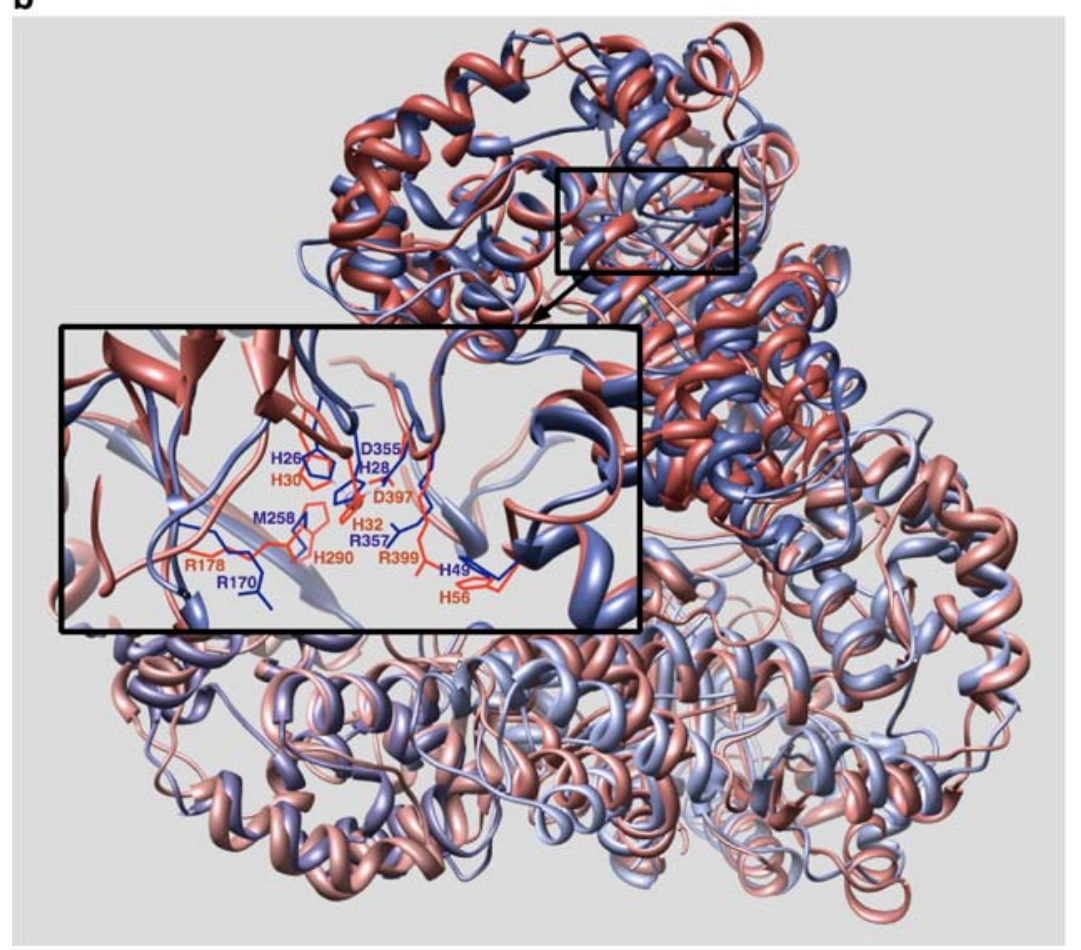


To gain further insight into the structural differences between Bh0493 and the "typical" uronate isomerases (and between uronate isomerases and other members of the amidohydrolase superfamily), and in the absence of a structure of $\mathrm{Bh} 0705$, we compared the structure of Bh0493 (PDB codes 2Q08 and 2Q6E) to another "typical" uronate isomerase from Thermotoga maritima (PDB code 1J5S). As shown in Fig. 4b, the functionally important residues Arg170, Arg357 and His49, are conserved and cluster together within the enzyme active site both in the T. maritima enzyme and Bh0493. However, an additional metal-coordinating histidine that is usually found at the end of $\beta$-strand five in "typical" uronate isomerases ( $\mathrm{H} 290$ in the $1 \mathrm{~J} 5 \mathrm{~S})$ is missing in Bh0493, which has a Met (M258) in that position. The $\mathrm{Zn}^{2+}$ ion is coordinated by two histidine residues (His28 and His26) plus Asp355. Guided by these structures, further biochemical and computational studies to examine the differences between these two types of uronate isomerases, and how they may be related to their different specificities, are currently in progress.

\section{Enolase superfamily example: mandelate racemase subgroup}

The SFLD currently describes 17 different families in the enolase superfamily, each performing a different overall reaction associated with different substrates and products. For the approximately $50 \%$ of the superfamily sequences whose functions are yet unknown, we estimate that roughly 15-20 novel functions (i.e. new families) will be identified. Across the superfamily, the sequences whose functions are not yet identified can be clustered into three primary subgroups and several smaller ones based on sequence and structural differences, including differences in the constellations of active site residues involved in binding specificity and catalysis [10]. In the mandelate racemase subgroup, most of the enzymes with characterized reactions are dehydratases acting on acid sugars, with the "outlier" enzyme being mandelate racemase itself. All structurally characterized members of the subgroup can be distinguished by a His-Asp dyad at the ends of $\beta$-strands six and seven that is associated with proton abstraction of substrates in the R-configuration [59]. Mandelate racemase and several acid sugar dehydratases that were previously structurally and functionally characterized also have a conserved Lys-X-Lys motif on $\beta$-strand two, with the second Lys in this motif involved in proton abstraction of substrates in the S-configuration [42]. Within this subgroup, we also observe divergence in this motif among several members of both known [32] and unknown function.
Three members of the mandelate racemase subgroup whose structures were determined by NYSGXRC, 2GL5 and 2056 from Salmonella typhimurium and 20X4 from Zymomonas mobilis, were found to have a Lys-Val-Asp sequence motif at this position, possibly indicating a different catalytic mechanism or yet other novel function(s). The three structures align within $50 \%$ sequence identity to each other. The next closest structures $(30 \%$ sequence identity) are also members of the mandelate racemase subgroup: 2POZ from Mesorhizobium loti and 2POD from Burkholderia pseudomallei have Lys-Phe-Tyr and Lys-Ile-Trp motifs at this position, respectively, providing further evidence for divergent catalytic function(s). Their structures reveal details of differences relative to that of well-characterized subgroup members containing a "canonical" Lys-X-Lys motif, providing information expected to be useful in identifying their functions. Figure 5 shows superpositions of mandelate racemase with $2 \mathrm{GL} 5$ and $2 \mathrm{POD}$, illustrating the differences in this motif. Guided by these new structures, these enzymes are now being further analyzed computationally and experimentally.

\section{Conclusion}

The Enzyme Specificity Consortium and the New York SGX Research Center for Structural Genomics made significant progress towards characterizing the structures and functions in the amidohydrolase and enolase superfamilies. New members of the amidohydrolase superfamily have been identified through a combination of sequence- and structure-based expansions of the pool of known superfamily members. The structure-based expansion was particularly successful in identifying previously unrecognized superfamily members. The 63 crystallographic structures from the structural genomics pipeline increased the fraction of the sequences in these two superfamilies that can be modeled based on at least $30 \%$ sequence identity from $45 \%$ to $73 \%$.

As an annotation tool for the targets in the two superfamilies, template-based modeling of all sequences that had detectable homology to a known structure in the amidohydrolase or enolase superfamily allowed us to suggest previously un-annotated amidohydrolase sequences, several of which were subsequently verified by experiment, as shown for Bh0493 in this paper. This demonstrates the power of combining sequenceand structure-based approaches for the structural genomics of two large and diverse enzyme superfamilies. 
Fig. 5 Mandelate racemase bound to a substrate analog, atrolactate, (1MDR: red), is shown superimposed with two structures of unknown function. In both superpositions, active site metal ligands D195, E221, E247, the active site His-Asp dyad (H297, D270), and a LysX-Lys motif (K164, K166) conserved in $1 \mathrm{MDR}$ and other members of the mandelate racemase subgroup are labeled (1MDR numbering). a Superposition of 2GL5 (blue) with $1 \mathrm{MDR}$ shows conservation of all of these active site residues, except for the second Lys in the Lys-X-Lys motif of $1 \mathrm{MDR}$, which is replaced in 2GL5 by Asp170. This residue faces away from the active site in 2GL5. b Superposition of 2POD (green) with 1MDR also shows conservation of all of listed residues, except for the second Lys in the Lys-X-Lys motif that is replaced in $2 \mathrm{POD}$ by W176
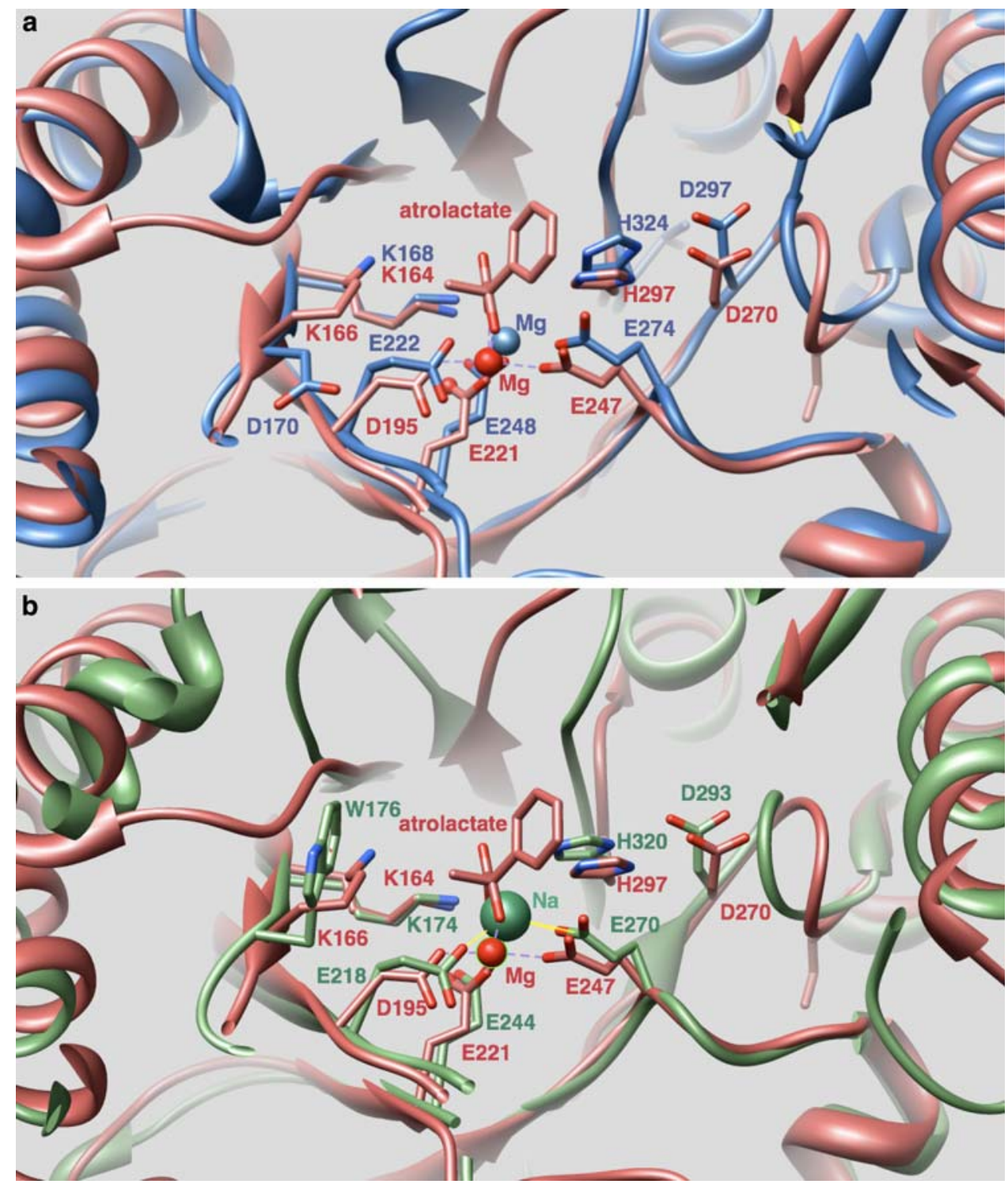

Acknowledgements This work was supported by NIH (U54 GM074945 (Principal Investigator: Stephen K. Burley) and P01 GM71790), the Sandler Family Supporting Foundation, Fight for Mike Foundation, Ron Conway, Hewlett-Packard, NetApp, IBM, and Intel. Use of the Advanced Photon Source was supported by the U.S. Department of Energy, Office of Basic Energy Sciences. Use of the SGX-CAT beam line facilities at Sector 31 of the APS was provided by SGX Pharmaceuticals, which constructed and operates the facility. Use of the NSLS beamline X29 was supported by the DOE and P41-EB-01979. Molecular graphics images were produced using the UCSF Chimera package from the Resource for Biocomputing, Visualization, and Informatics at the University of California, San Francisco (supported by NIH P41 RR-01081).

Open Access This article is distributed under the terms of the Creative Commons Attribution Noncommercial License which permits any noncommercial use, distribution, and reproduction in any medium, provided the original author(s) and source are credited.

\section{References}

1. Andreeva A, Murzin AG (2006) Evolution of protein fold in the presence of functional constraints. Curr Opin Struct Biol 16:399_ 408

2. Gerlt JA, Babbitt PC (2001) Divergent evolution of enzymatic function: mechanistically diverse superfamilies and functionally distinct suprafamilies. Annu Rev Biochem 70:209-246

3. Glasner ME, Gerlt JA, Babbitt PC (2006) Evolution of enzyme superfamilies. Curr Opin Chem Biol 10:492-497

4. Todd AE, Orengo CA, Thornton JM (2001) Evolution of function in protein superfamilies, from a structural perspective. J Mol Biol 307:1113-1143

5. Pegg SC, Brown S, Ojha S, Huang CC, Ferrin TE, Babbitt PC et al (2005) Representing structure-function relationships in mechanistically diverse enzyme superfamilies. Pac Symp Biocomput 358-369

6. Holm L, Sander C (1997) An evolutionary treasure: unification of a broad set of amidohydrolases related to urease. Proteins 28: $72-82$ 
7. Seibert CM, Raushel FM (2005) Structural and catalytic diversity within the amidohydrolase superfamily. Biochemistry 44:63836391

8. Pegg SC, Brown SD, Ojha S, Seffernick J, Meng EC, Morris JH, Chang PJ, Huang CC, Ferrin TE, Babbitt PC (2006) Leveraging enzyme structure-function relationships for functional inference and experimental design: the structure-function linkage database. Biochemistry 45:2545-2555

9. Babbitt PC, Hasson MS, Wedekind JE, Palmer DR, Barrett WC, Reed GH, Rayment I, Ringe D, Kenyon GL, Gerlt JA (1996) The enolase superfamily: a general strategy for enzyme-catalyzed abstraction of the alpha-protons of carboxylic acids. Biochemistry 35:16489-16501

10. Gerlt JA, Babbitt PC, Rayment I (2005) Divergent evolution in the enolase superfamily: the interplay of mechanism and specificity. Arch Biochem Biophys 433:59-70

11. Vick JE, Gerlt JA (2007) Evolutionary potential of (beta/alpha)8barrels: stepwise evolution of a "new" reaction in the enolase superfamily. Biochemistry 46:14589-14597

12. Akana J, Fedorov AA, Fedorov E, Novak WR, Babbitt PC, Almo SC, Gerlt JA (2006) D-Ribulose 5-phosphate 3-epimerase: functional and structural relationships to members of the ribulosephosphate binding (beta/alpha)8-barrel superfamily. Biochemistry 45:2493-2503

13. Almo SC, Bonanno JB, Sauder JM, Emtage S, Dilorenzo TP, Malashkevich V, Wasserman SR, Swaminathan S, Eswaramoorthy S, Agarwal R, Kumaran D, Madegowda M, Ragumani S, Patskovsky Y, Alvarado J, Ramagopal UA, Faber-Barata J, Chance MR, Sali A, Fiser A, Zhang ZY, Lawrence DS, Burley SK (2007) Structural genomics of protein phosphatases. J Struct Funct Genomics 8:121-140

14. Bonanno JB, Almo SC, Bresnick A, Chance MR, Fiser A, Swaminathan S, Jiang J, Studier FW, Shapiro L, Lima CD, Gaasterland TM, Sali A, Bain K, Feil I, Gao X, Lorimer D, Ramos A, Sauder JM, Wasserman SR, Emtage S, D'Amico KL, Burley SK (2005) New York-Structural GenomiX Research Consortium (NYSGXRC): a large scale center for the protein structure initiative. J Struct Funct Genomics 6:225-232

15. Brown SD, Gerlt JA, Seffernick JL, Babbitt PC (2006) A gold standard set of mechanistically diverse enzyme superfamilies. Genome Biol 7:R8

16. Gerlt JA (2007) A protein structure (or function?) initiative. Structure 15:1353-1356

17. Glasner ME, Fayazmanesh N, Chiang RA, Sakai A, Jacobson MP, Gerlt JA, Babbitt PC (2006) Evolution of structure and function in the o-succinylbenzoate synthase/ $\mathrm{N}$-acylamino acid racemase family of the enolase superfamily. J Mol Biol 360:228-250

18. Glasner ME, Gerlt JA, Babbitt PC (2007) Mechanisms of protein evolution and their application to protein engineering. Adv Enzymol Relat Areas Mol Biol 75:193-239 xii-xiii

19. Hall RS, Brown S, Fedorov AA, Fedorov EV, Xu C, Babbitt PC, Almo SC, Raushel FM (2007) Structural diversity within the mononuclear and binuclear active sites of N-acetyl-D-glucosamine-6-phosphate deacetylase. Biochemistry 46:7953-7962

20. Hermann JC, Ghanem E, Li Y, Raushel FM, Irwin JJ, Shoichet BK (2006) Predicting substrates by docking high-energy intermediates to enzyme structures. J Am Chem Soc 128:1588215891

21. Hermann JC, Marti-Arbona R, Fedorov AA, Fedorov E, Almo SC, Shoichet BK, Raushel FM (2007) Structure-based activity prediction for an enzyme of unknown function. Nature 448: 775-779

22. Imker HJ, Fedorov AA, Fedorov EV, Almo SC, Gerlt JA (2007) Mechanistic diversity in the RuBisCO superfamily: the "enolase" in the methionine salvage pathway in Geobacillus kaustophilus. Biochemistry 46:4077-4089
23. Irwin JJ, Raushel FM, Shoichet BK (2005) Virtual screening against metalloenzymes for inhibitors and substrates. Biochemistry 44:12316-12328

24. Li Y, Raushel FM (2005) Inhibitors designed for the active site of dihydroorotase. Bioorg Chem 33:470-483

25. Liao RZ, Yu JG, Raushel FM, Himo F (2008) Theoretical investigation of the reaction mechanism of the dinuclear zinc enzyme dihydroorotase. Chemistry 14:4287-4292

26. Marti-Arbona R, Raushel FM (2006) Mechanistic characterization of $\mathrm{N}$-formimino-L-glutamate iminohydrolase from Pseudomonas aeruginosa. Biochemistry 45:14256-14262

27. Marti-Arbona R, Thoden JB, Holden HM, Raushel FM (2005) Functional significance of Glu-77 and Tyr-137 within the active site of isoaspartyl dipeptidase. Bioorg Chem 33:448-458

28. Marti-Arbona R, Xu C, Steele S, Weeks A, Kuty GF, Seibert CM, Raushel FM (2006) Annotating enzymes of unknown function: $\mathrm{N}$-formimino-L-glutamate deiminase is a member of the amidohydrolase superfamily. Biochemistry 45:1997-2005

29. Nguyen TT, Brown S, Fedorov AA, Fedorov EV, Babbitt PC, Almo SC, Raushel FM (2008) At the periphery of the amidohydrolase superfamily: Bh0493 from Bacillus halodurans catalyzes the isomerization of D-galacturonate to D-tagaturonate. Biochemistry 47:1194-1206

30. Nowlan C, Li Y, Hermann JC, Evans T, Carpenter J, Ghanem E, Shoichet BK, Raushel FM (2006) Resolution of chiral phosphate, phosphonate, and phosphinate esters by an enantioselective enzyme library. J Am Chem Soc 128:15892-15902

31. Porter TN, Li Y, Raushel FM (2004) Mechanism of the dihydroorotase reaction. Biochemistry 43:16285-16292

32. Rakus JF, Fedorov AA, Fedorov EV, Glasner ME, Vick JE, Babbitt PC, Almo SC, Gerlt JA (2007) Evolution of enzymatic activities in the enolase superfamily: D-Mannonate dehydratase from Novosphingobium aromaticivorans. Biochemistry 46: 12896-12908

33. Sakai A, Xiang DF, Xu C, Song L, Yew WS, Raushel FM, Gerlt JA (2006) Evolution of enzymatic activities in the enolase superfamily: N-succinylamino acid racemase and a new pathway for the irreversible conversion of D- to L-amino acids. Biochemistry 45:4455-4462

34. Song L, Kalyanaraman C, Fedorov AA, Fedorov EV, Glasner ME, Brown S, Imker HJ, Babbitt PC, Almo SC, Jacobson MP, Gerlt JA (2007) Prediction and assignment of function for a divergent $\mathrm{N}$-succinyl amino acid racemase. Nat Chem Biol 3:486-491

35. Thoden JB, Taylor Ringia EA, Garrett JB, Gerlt JA, Holden HM, Rayment I (2004) Evolution of enzymatic activity in the enolase superfamily: structural studies of the promiscuous $o$-succinylbenzoate synthase from Amycolatopsis. Biochemistry 43:5716-5727

36. Tyagi R, Eswaramoorthy S, Burley SK, Raushel FM, Swaminathan $S$ (2008) A common catalytic mechanism for proteins of the HutI family. Biochemistry 47:5608-5615

37. Vick JE, Schmidt DM, Gerlt JA (2005) Evolutionary potential of (beta/alpha)8-barrels: in vitro enhancement of a "new" reaction in the enolase superfamily. Biochemistry 44:11722-11729

38. Weeks A, Lund L, Raushel FM (2006) Tunneling of intermediates in enzyme-catalyzed reactions. Curr Opin Chem Biol $10: 465-472$

39. Williams L, Nguyen T, Li Y, Porter TN, Raushel FM (2006) Uronate isomerase: a nonhydrolytic member of the amidohydrolase superfamily with an ambivalent requirement for a divalent metal ion. Biochemistry 45:7453-7462

40. Yew WS, Fedorov AA, Fedorov EV, Rakus JF, Pierce RW, Almo SC, Gerlt JA (2006) Evolution of enzymatic activities in the enolase superfamily: L-fuconate dehydratase from Xanthomonas campestris. Biochemistry 45:14582-14597 
41. Yew WS, Fedorov AA, Fedorov EV, Wood BM, Almo SC, Gerlt JA (2006) Evolution of enzymatic activities in the enolase superfamily: D-tartrate dehydratase from Bradyrhizobium japonicum. Biochemistry 45:14598-14608

42. Yew WS, Fedorov AA, Fedorov EV, Almo SC, Gerlt JA (2007) Evolution of enzymatic activities in the enolase superfamily: Ltalarate/galactarate dehydratase from Salmonella typhimurium LT2. Biochemistry 46:9564-9577

43. Norvell JC, Berg JM (2007) Update on the protein structure initiative. Structure 15:1519-1522

44. Berman H, Henrick K, Nakamura H, Markley JL (2007) The worldwide Protein Data Bank (wwPDB): ensuring a single, uniform archive of PDB data. Nucleic Acids Res 35:D301-D303

45. Eswar N, John B, Mirkovic N, Fiser A, Ilyin VA, Pieper U, Stuart AC, Marti-Renom MA, Madhusudhan MS, Yerkovich B, Sali A (2003) Tools for comparative protein structure modeling and analysis. Nucleic Acids Res 31:3375-3380

46. Wu CH, Apweiler R, Bairoch A, Natale DA, Barker WC, Boeckmann B, Ferro S, Gasteiger E, Huang H, Lopez R, Magrane M, Martin MJ, Mazumder R, O’Donovan C, Redaschi N, Suzek B (2006) The Universal Protein Resource (UniProt): an expanding universe of protein information. Nucleic Acids Res 34:D187-D191

47. Altschul SF, Madden TL, Schaffer AA, Zhang J, Zhang Z, Miller W, Lipman DJ (1997) Gapped BLAST and PSI-BLAST: a new generation of protein database search programs. Nucleic Acids Res 25:3389-3402

48. Pieper U, Eswar N, Davis FP, Braberg H, Madhusudhan MS, Rossi A, Marti-Renom M, Karchin R, Webb BM, Eramian D, Shen MY, Kelly L, Melo F, Sali A (2006) MODBASE: a database of annotated comparative protein structure models and associated resources. Nucleic Acids Res 34:D291-D295

49. Venter JC, Remington K, Heidelberg JF, Halpern AL, Rusch D, Eisen JA, Wu D, Paulsen I, Nelson KE, Nelson W, Fouts DE, Levy S, Knap AH, Lomas MW, Nealson K, White O, Peterson J, Hoffman J, Parsons R, Baden-Tillson H, Pfannkoch C, Rogers YH, Smith HO (2004) Environmental genome shotgun sequencing of the Sargasso Sea. Science 304:66-74

50. Lee J, Ware B (2003) Open source web development with LAMP: using Linux, Apache, MySQL, Per, and PHP. AddisonWesley, Boston

51. Shannon P, Markiel A, Ozier O, Baliga NS, Wang JT, Ramage D, Amin N, Schwikowski B, Ideker T (2003) Cytoscape: a software environment for integrated models of biomolecular interaction networks. Genome Res 13:2498-2504

52. Benson DA, Karsch-Mizrachi I, Lipman DJ, Ostell J, Wheeler DL (2008) GenBank. Nucleic Acids Res 36:D25-D30

53. Atkinson HJ, Morris JH, Ferrin TE, Babbitt PC et al (2009) Using sequence similarity networks for visualization of relationships across diverse protein superfamilies. PLoS One (submitted)

54. Martin AC, MacArthur M W, Thornton JM et al (1997) Assessment of comparative modeling in CASP2. Proteins, Suppl $1: 14-28$

55. Sanchez R, Sali A (1997) Advances in comparative proteinstructure modelling. Curr Opin Struct Biol 7:206-214

56. Vitkup D, Melamud E, Moult J, Sander C (2001) Completeness in structural genomics. Nat Struct Biol 8:559-566

57. Nagano N, Orengo CA, Thornton JM (2002) One fold with many functions: the evolutionary relationships between TIM barrel families based on their sequences, structures and functions. J Mol Biol 321:741-765

58. Gough J, Karplus K, Hughey R, Chothia C (2001) Assignment of homology to genome sequences using a library of hidden Markov models that represent all proteins of known structure. J Mol Biol 313:903-919

59. Schafer SL, Barrett WC, Kallarakal AT, Mitra B, Kozarich JW, Gerlt JA, Clifton JG, Petsko GA, Kenyon GL (1996) Mechanism of the reaction catalyzed by mandelate racemase: structure and mechanistic properties of the D270 N mutant. Biochemistry 35:5662-5669

60. Melo F, Sanchez R, Sali A (2002) Statistical potentials for fold assessment. Protein Sci 11:430-448

61. Wheeler DL, Barrett T, Benson DA, Bryant SH, Canese K, Chetvernin V, Church DM, Dicuccio M, Edgar R, Federhen S, Feolo M, Geer LY, Helmberg W, Kapustin Y, Khovayko O, Landsman D, Lipman DJ, Madden TL, Maglott DR, Miller V, Ostell J, Pruitt KD, Schuler GD, Shumway M, Sequeira E, Sherry ST, Sirotkin K, Souvorov A, Starchenko G, Tatusov RL, Tatusova TA, Wagner L, Yaschenko E (2008) Database resources of the National Center for Biotechnology Information. Nucleic Acids Res 36:D13-D21

62. Pettersen EF, Goddard TD, Huang CC, Couch GS, Greenblatt DM, Meng EC, Ferrin TE (2004) UCSF Chimera-a visualization system for exploratory research and analysis. J Comput Chem 25:1605-1612 